\title{
La construction identitaire de la patria dans le discours des États de Languedoc (1346-1484)
}

Sylvie Quéré

\section{OpenEdition}

\section{Journals}

Édition électronique

URL : http://journals.openedition.org/ifha/8040

DOI : 10.4000/ifha. 8040

ISSN : 2198-8943

\section{Éditeur}

IFRA - Institut franco-allemand (sciences historiques et sociales)

\section{Édition imprimée}

Date de publication : 31 décembre 2014

ISSN : 2190-0078

\section{Référence électronique}

Sylvie Quéré, "La construction identitaire de la patria dans le discours des États de Languedoc (1346-1484) », Revue de l'IFHA [En ligne], 6 | 2014, mis en ligne le 31 décembre 2014, consulté le 03 mai 2019. URL : http://journals.openedition.org/ifha/8040 ; DOI : 10.4000/ifha.8040

Ce document a été généré automatiquement le 3 mai 2019

(CIFHA 


\section{La construction identitaire de la patria dans le discours des États de Languedoc (1346-1484)}

\section{Sylvie Quéré}

Cette communication s'inscrit dans les suites d'une thèse de doctorat ${ }^{1}$ dont l'objectif visait à mettre en évidence les fondements et l'évolution du discours politique des États de Languedoc à la fin du Moyen Âge. Une telle évolution ne pouvant être observée que sur une durée relativement longue, notre recherche couvrait une période de près d'un siècle et demi, soit de 1346 à 1484 . Nous l'avons bornée, en amont, par la deuxième réunion des États de Languedoc ${ }^{2}$ et, en aval, par la tenue des États généraux de Tours. Nous l'avons découpée en quatre périodes, ce qui nous a permis d'observer l'évolution du discours, d'en révéler les continuités et d'en repérer les ruptures. Identifiées respectivement par les lettres $\mathrm{A}$ à $\mathrm{D}$ (voir tableau 1), ces quatre périodes étaient fondées sur les rapports de force que les États de Languedoc avaient entretenus avec la royauté et conséquemment l'importance des droits qu'ils avaient pu conséquemment exercer.

Tableau 1 : Définition des périodes de la recherche

\begin{tabular}{|l|l|l|}
\hline période & date de début & date de fin \\
\hline A & 1346 & 1380 \\
\hline B & 1381 & 1416 \\
\hline C & 1417 & 1442 \\
\hline D & 1443 & 1484 \\
\hline
\end{tabular}


Nous ne nous étendrons pas ici sur les aspects méthodologiques de cette recherche, mais nous aimerions en souligner les éléments les plus importants. Notre problématique s'articulait autour de trois hypothèses fondamentales. En premier lieu, contrairement à ce qu'avait affirmé leur principal historien, Henri Gilles ${ }^{3}$, les États de Languedoc ont joué un rôle politique effectif à la fin du Moyen Âge, c'est-à-dire qu'au-delà de leurs fonctions strictement économique, juridique et fiscale, ils ont su établir et maintenir avec le prince un dialogue basé sur leur prétention à représenter le "pays" et à en assumer collectivement les devoirs et les droits. En deuxième lieu, dans le cadre de ce dialogue, les États ont tenu un discours politique par lequel ils ont cherché à imposer au prince leurs propres représentations de l'État et de leur rôle au sein de ce dernier. Enfin, qu'à l'inverse de ce qu'avait également déploré Henri Gilles, ces représentations pouvaient se révéler dans le discours des États à travers les actes ${ }^{4}$ qu'ils ont laissés, à condition d'adopter une méthodologie qui puisse en transcender le côté purement institutionnel.

Pour mener à bien cette recherche, nous avons composé un corpus répondant aux critères usuels de constitution - homogénéité, contrastivité et diachronicité ${ }^{5}$-, c'est-àdire un ensemble de textes qui : 1) sans être identiques dans leur constitution, sont quasi exhaustifs dans le champ discursif étudié et peuvent être assignés à des situations de communication déterminées ; 2) permettent la comparaison avec d'autres discours, de façon à repérer les discordances; 3) s'échelonnent dans le temps, afin d'en mettre en évidence les continuités et les ruptures. Composé de 326 actes, émanant directement des États ou du pouvoir royal, ce corpus contenait, sous forme d'ordonnances, de cahiers ou de lettres, la plupart des requêtes faites au roi par les États de Languedoc et les réponses données par le roi à ces mêmes requêtes en échange du consentement à l'impôt. Par leur forme diplomatique, où texte et contexte se retrouvent dans un même espace construit, ces actes pouvaient nous fournir une grande partie des ressources nécessaires à l'interprétation du discours.

Nous avons traité ce corpus à l'aide d'une méthodologie inspirée des travaux de l'école française d'analyse du discours, basés sur des démarches propres à l'analyse du discours, à la pragmatique et à la linguistique des actes de parole. Nous l'avons toutefois fait avec une grande liberté, en raison des difficultés inhérentes à l'exploitation d'un corpus constitué majoritairement de sources manuscrites, de surcroît rédigées en trois langues différentes ${ }^{6}$, et dont chacune comporte des variations lexicales importantes. Notre méthode s'est ainsi fondée sur une analyse d'énoncés tournant autour d'un certain nombre de mots pivots correspondant à différents champs sémantiques, analyse rendue possible par l'élaboration d'une grille de catégorisation sociosémantique et la constitution d'une base de données lexicale contenant 2741 énoncés et plus de 60000 occurrences de mots ou expressions ${ }^{7}$. C'est donc munie de cet appareillage conceptuel et méthodologique que nous avons procédé à l'analyse du système de représentation par lequel les États de Languedoc ont cherché à légitimer leur identité sociale et leur existence au sein de la société politique.

Cette recherche nous a notamment menée à nous pencher sur la question de la nation, et plus spécifiquement sur l'émergence dans le discours politique des États de Languedoc, au cours du premier tiers du XVe siècle, du concept de patria en tant qu'assise de la construction identitaire d'un "discours régionaliste", au sens où l'a défini Pierre Bourdieu, c'est-à-dire « un discours performatif, visant à imposer comme légitime une nouvelle définition des frontières et à faire connaître et reconnaitre la région ainsi 
délimitée contre la définition dominante et méconnue comme telle, donc reconnue et légitime, qui l'ignore ${ }^{8}$.

Nous avons ainsi pu démontrer que le discours des États de Languedoc montrait une évolution des représentations sur le pays par lesquelles la patria, définie d'abord par ses parties constitutives ${ }^{9}$ (voir tableau 2), avait acquis une existence propre entre 1417 et 1430 , alors que l'expression "pays de Languedoc » devient courante au sein du discours des États (voir tableau 3). Le "pays de Languedoc », en tant qu'entité régionale, semble ainsi s'imposer durant les années tumultueuses qui voient successivement Charles de Ponthieu devenir lieutenant général du royaume ${ }^{10}$, se réfugier à Bourges après la signature du traité de Troyes, et revendiquer le trône de France à la suite de la mort de son père en 1422. À cet égard, rappelons ici que les États de Languedoc, après avoir brièvement soutenu les Bourguignons, se sont rangés assez tôt derrière le Dauphin, leur « naturel » seigneur.

Tableau 2 : Occurrences des noms relatifs aux parties constitutives du pays

\begin{tabular}{|l|l|l|l|l|l|}
\hline nom & A & B & C & D & total \\
\hline sénéchaussée & 36 & 6 & 17 & 29 & 88 \\
\hline diocèse & 1 & 1 & 17 & 48 & 67 \\
\hline partie & 10 & 1 & 2 & 6 & 19 \\
\hline contrée & & & & 13 & 13 \\
\hline province & 1 & & 1 & 1 & 3 \\
\hline total & 48 & 8 & 37 & 97 & 190 \\
\hline
\end{tabular}

Tableau 3 : Occurrences des expressions relatives à la désignation du pays

\begin{tabular}{|l|l|l|l|l|l|}
\hline expression & A & B & C & D & total \\
\hline pays & 21 & 10 & 152 & 566 & 749 \\
\hline Languedoc & 17 & 1 & 85 & 227 & 330 \\
\hline trois sénéchaussées & 2 & 8 & 6 & 2 & 18 \\
\hline parties occitanes & 4 & & 3 & & 7 \\
\hline par deçà/delà & & & 4 & 2 & 6 \\
\hline total & 44 & 19 & 250 & 797 & 1110 \\
\hline
\end{tabular}

Par ailleurs, au-delà des noms par lesquels les États désignent le pays, les verbes auxquels se rattache directement cette notion nous ont fourni certains indices quant aux représentations que se font les États du pays. En examinant la liste des verbes utilisés par 
les États, nous avons constaté que ceux-ci font apparaître trois représentations différentes du pays en tant qu'espace géographique. Celle, d'abord, d'un espace de vie où l'on peut «vivre", « habiter » et « demeurer ». C'est le pays natal. Celle, ensuite, d'un espace de mouvement, où l'on peut «aller » et « venir », « entrer » et "saillir ». C'est le pays marchand, mais aussi le pays religieux ou militaire, par lequel passent les pèlerins et les gens de guerre. Celle, finalement, d'un espace ouvert, mais limité, distinct des autres pays ou seigneuries "estranges" qui, au-delà des «limites" et des "frontières", « marchent » avec le pays de Languedoc. C'est le pays politique, soumis à l'autorité du roi de France. Il est intéressant de noter que nous n'avons trouvé dans notre corpus aucun verbe relatif à la langue, tel «parler ", qui aurait pu témoigner d'une représentation du pays en tant qu'espace linguistique. De toute évidence, la langue occitane, bien qu'elle ait donné son nom à cette région, n'apparait pas liée aux diverses représentations du pays véhiculées dans le discours des États de Languedoc.

Cet espace géographique qu'est le "pays de Languedoc » se définit également par ses privilèges, rappelés à maintes reprises par les États (voir tableau 4), principalement le droit écrit, la liberté de marchandise et le consentement à l'impôt. Ces énoncés participent en effet à la construction identitaire du pays dans la mesure où « tout énoncé sur la région fonctionne comme un argument qui contribue à favoriser ou à défavoriser l'accès de la région à la reconnaissance et, par là, à l'existence ${ }^{11}$ ». Il est vrai que ce discours est loin d'être propre au Languedoc puisqu'il s'agit d'un topos employé par presque toutes les régions du royaume. Pierre-Jean Souriac a ainsi affirmé que le «lien fiscal entre le souverain et ses sujets ne représente pas une spécificité particulière du Languedoc, car chaque province du royaume était soumise au même régime et entretenait probablement un dialogue similaire avec son souverain, du moins celles dotées d'une assemblée d'États ${ }^{12}$ ». Mais ce qui «fait» le pays de Languedoc est la combinaison unique de ces privilèges, leur défense par les États et leur maintien - voire leur renforcement - bien au-delà du milieu du XVe siècle. Dans le discours des États, la patria se révèle donc comme un espace géographique, à la fois pays natal, pays marchand et pays politique, doté de privilèges distincts, ou plutôt, devrions-nous dire, d'une combinaison unique de privilèges qui « font » le pays.

Tableau 4 : Occurrences des noms relatifs aux libertés du pays

\begin{tabular}{|l|l|l|l|l|l|}
\hline nom & A & $B$ & $C$ & $D$ & total \\
\hline liberté & 13 & 1 & 5 & 66 & 85 \\
\hline franchise & 6 & 1 & 4 & 51 & 62 \\
\hline privilège & 10 & 2 & & 45 & 57 \\
\hline coutume & 7 & 1 & 3 & 14 & 25 \\
\hline usage & 8 & 1 & 1 & 7 & 17 \\
\hline ordonnance & 1 & & & 11 & 12 \\
\hline droit & 4 & & & 4 & 8 \\
\hline
\end{tabular}




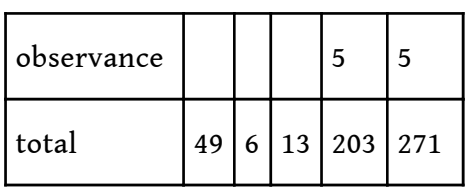

De surcroît, ce pays forme un «corps ${ }^{13}$ », parlant d' « une seule voix » et dont les États, unis dans la défense de ses privilèges, constituent à la fois l'« âme » et le "coeur ». La période comprise entre 1417 et 1430 est ainsi celle où les " trois états ${ }^{14}$ ", qui se désignent désormais sous cette appellation en tant qu'entité collective (voir tableau 5), commencent à parler véritablement au nom de l'ensemble des habitants du pays (voir tableau 6). À travers les différents rôles qu'ils assument en tant que représentants légitimes du pays exposer, consentir, demander, ordonner, protester et conseiller ${ }^{15}$-, les États de Languedoc se présentent alors comme "fossan d'un cor et d'una volontat de gardar et de deffendre lor e-l pays dels enemys ${ }^{16}$ ", et ce, afin que les trois sénéchaussées "se puescan gardar et deffendre et adjudar la hun a l'autre ${ }^{17}$ ». De la même façon, le projet d'union de mai 1430 montre les trois états formant «unum cor, una anima, una affectio ad publicam utilitatem ${ }^{18}$ ». Et si les États sont "un coeur", c'est parce qu'ils forment "un corps", comme on le trouve très clairement exprimé en 1419 dans l'octroi fait par les États où " las gens ordenatz en lodit conseilh, representan hun cors de las dichas tres senescalcias, offren de far tenir et servar et pagar las causas et soma dessus ditas ${ }^{19}$ ». La notion de " corps » revient également dans le cahier de 1479, alors que les États demandent que la part et portion de plusieurs paroisses et villages, "lesquelz ont este desmembrez et separés du corps du pays \& dioceses ${ }^{20} "$ ", soit retranchée de la contribution du pays.

Tableau 5 : Occurrences des expressions relatives à la désignation des États

\begin{tabular}{|l|l|l|l|l|l|}
\hline expression & A & B & C & D & total \\
\hline trois états & 6 & & 83 & 157 & 246 \\
\hline états & & & & 152 & 152 \\
\hline gens des trois états & & 38 & 114 & 152 \\
\hline total & 6 & 121 & 423 & 550 \\
\hline
\end{tabular}

Tableau 6 : Occurrences des noms relatifs aux habitants du pays

\begin{tabular}{|l|l|l|l|l|l|}
\hline nom & $A$ & $B$ & $C$ & $D$ & total \\
\hline habitants & 2 & & 19 & 138 & 159 \\
\hline peuple & & 1 & 23 & 108 & 132 \\
\hline sujets & 2 & & 13 & 74 & 89 \\
\hline total & 4 & 1 & 55 & 320 & 380 \\
\hline
\end{tabular}


Cette voix du pays, exprimée par les États, se manifeste principalement à travers un dialogue avec le prince, perçu par les États de Languedoc comme leur «naturel » et « souverain » seigneur (voir tableaux 7 et 8 ). En ce domaine, il serait difficile de trouver un discours aussi empreint de loyauté, d'amour et d'obéissance que celui des États de Languedoc envers les rois de France. Le sentiment politique y est partout, sinon clairement exprimé, du moins sous-entendu. Le discours des États constitue ainsi une manifestation éloquente de la fidélité des Languedociens envers les Valois, au demeurant caractéristique des villes du Midi ${ }^{21}$ où l'amour et l'obéissance vont également de pair ${ }^{22}$. En définitive, Philippe Contamine a assurément trouvé la formule la plus juste en parlant de la « fidélité éclairée, à l'occasion critique ${ }^{23}$ » des États de Languedoc et Patrick Arabeyre a certainement eu raison d'affirmer que "bien loin de contester la monarchie, les Méridionaux développeront aux XVe et XVIe siècles la mystique du pouvoir monarchique ${ }^{24}$ ». Au point où, pour quiconque ignorerait la réalité du royaume de France à la fin du Moyen Âge, la lecture des actes des États pourrait laisser croire que les Valois ne sont pas les rois de France, Rex Franciae, mais bien les rois du pays de Languedoc, Rex patriae.

Tableau 7 : Occurrences de l'adjectif « naturel » relatives à la personne du roi

\begin{tabular}{|l|l|l|l|l|l|}
\hline adjectif & $A$ & $B$ & $C$ & $D$ & total \\
\hline naturel & & & 4 & 50 & 54 \\
\hline
\end{tabular}

Tableau 8 : Occurrences de l'adjectif « souverain » dans la désignation du roi

\begin{tabular}{|l|l|l|l|l|l|}
\hline adjectif & A & B & C & D & total \\
\hline souverain & & & 23 & 55 & 78 \\
\hline
\end{tabular}

En résumé, notre recherche a montré que le discours identitaire des États de Languedoc et partant, celui de la patria - s'était construit entre 1417 et 1430, alors que ces derniers commencent à parler au nom de l'ensemble des habitants du pays et que sont établis les caractères qui le « disent ». Mais, en lien avec le thème du colloque, que peut-on conclure de l'analyse de ces représentations du pays quant à la possible existence d'un sentiment national proprement languedocien? Selon Philippe Contamine, ce sentiment n'existerait tout simplement pas. La construction du pays résulterait en effet d'une initiative monarchique et se confondrait, d'un point de vue institutionnel et politique, avec ses trois circonscriptions administratives. En fin de compte, le pays de Languedoc se définirait davantage par une communauté d'intérêts que par un sentiment national ${ }^{25}$. À quelques nuances près, cette vision est également celle de Philippe Wolff ${ }^{26}$ et d'Henri Bresc $^{27}$.

Or, si nous nous fions à la propre définition de Philippe Contamine, non seulement la communauté d'intérêts ne s'opposerait pas au sentiment national, mais elle en constituerait même l'un des fondements :

[le sentiment national correspond] à la fois à l'attachement vécu, instinctif, «naturel », envers un pays, une terre, et au sentiment qu'ils ont quelque chose en commun avec tous ceux qui y vivent et surtout qui y sont nés, autrement dit qu'il 
existe entre eux nécessairement une certaine solidarité, une communauté d'origine et de destin ${ }^{28}$

Partant de ce fait, sans remettre en cause la thèse de Philippe Contamine sur la construction monarchique du Languedoc au XIIIe siècle, ne pourrait-on pas envisager que cette communauté d'intérêts ait pu contribuer au développement des États de Languedoc et partant, à la création d'un lieu d'expression d'un discours identitaire sur le pays ? À cet égard, Thomas N. Bisson a déjà souligné l'importance dans l'émergence d'une "growing consciousness of local and corporate privileges ${ }^{29}$ " des assemblées de Languedoc du XIIIe siècle, et plus particulièrement de la représentation des villes aux différentes assemblées de sénéchaussées, dont les États sont devenus par la suite les principaux défenseurs. Par ailleurs, s'il faut en croire Léonard Dauphant, le sentiment «national » qui se construit autour de l'organisation royale de l'espace dans le royaume de France à la fin du Moyen Âge n'exclurait pas l'émergence d'une identité politique locale ${ }^{30}$. Selon ce dernier, l'échelon national voisinerait ainsi avec la conscience d'appartenir à une communauté locale, dans le cadre d'un pays (pagus) ou d'une ville, et, parfois également, avec une principauté régionale. De fait, c'est à l'échelle du pays qu'il faut chercher en Languedoc l'existence d'un sentiment national. Car, pour les États de Languedoc, le « royaume de France » ne représente en effet rien d'autre qu'un simple amalgame de pays, envers lequel ils n'éprouvent aucun attachement. Sans doute alors serait-il plus juste de parler de sentiment «patriotique» pour décrire cette conscience qu'éprouvent les Languedociens d'appartenir à une même patria.

Finalement, selon l'argumentaire scientifique du congrès, l'identité nationale pourrait être « une conscience propre et collective qui, d'une manière ou d'une autre, relève toujours d'une combinatoire associant communauté, territoire, autorité et culture ». Dès lors, pour répondre à notre question initiale, nous trouvons effectivement dans le discours des États de Languedoc l'expression d'une identité régionale "patriotique ", forcément collective, fondée à la fois sur les particularités du pays - le droit de consentir à l'impôt, le droit écrit, la liberté de marchandise, etc. -, son incarnation par les États et son alliance avec la royauté française depuis le XIIIe siècle.

\section{NOTES}

1. Sylvie Quéré, "Le discours politique des États de Languedoc à la fin du Moyen Âge (1346-1484 ", thèse présentée et soutenue publiquement le 19 octobre 2012, Montréal : Université du Québec à Montréal, 2012. En ligne: <http://www.archipel.uqam.ca/5240/1/D2393.pdf>. Consulté le 24 juin 2014.

2. La première réunion des États de Languedoc, qui eut lieu en janvier 1319, demeure un évènement isolé durant la première moitié du XIVe siècle.

3. Henri Gilles, Les États de Languedoc au XVe siècle, Toulouse : Privat, 1965.

4. Nous prenons ici le mot «acte» dans son sens le plus large d'une mise par écrit d'une action, et non dans le sens restreint d'un document à caractère juridique visé par une autorité reconnue. 
5. Voir Jacques Guilhaumou, «Le corpus en analyse de discours : perspective historique », Corpus et recherches linguistiques, $\mathrm{n}^{\circ} 1-2002$, novembre 2002, [s.p.]. En ligne : <http://corpus.revues.org/ document8.html>. Consulté le 24 juin 2014.

6. Soit 197 actes rédigés principalement en français, 112 en latin et 17 en occitan.

7. Cette base de données a été constituée à l'aide du logiciel FileMaker Pro.

8. Pierre Bourdieu, Ce que parler veut dire. L'économie des échanges linguistiques, Paris : Fayard, 1982, p. 137-138.

9. Rappelons qu'après le traité de Brétigny, le pays de Languedoc ne regroupe plus que les trois sénéchaussées de Toulouse, Beaucaire et Carcassonne.

10. Plus précisément le 14 juin 1417.

11. Pierre Bourdieu, Ce que parler veut dire, op. cit., p. 144.

12. Pierre-Jean Souriac, « Les états de Languedoc face à la guerre dans la première moitié du XVIe siècle", Cahiers de la Méditerranée, vol.71, 2005, paragraphe 25. En ligne: <http:// cdlm.revues.org/index946.html>. Consulté le 24 juin 2014.

13. Sur cette question, voir entre autres Barbara Stollberg-Rilinger, "La communication symbolique à l'époque pré-moderne. Concepts, thèses, perspectives de recherche ", Trivium Revue franco-allemande de sciences humaines et sociales, vol.2, 2008, [s.p.]. En ligne: <http:// trivium.revues.org/1152>. Consulté le 24 juin 2014.

14. Nous trouvons l'expression pour la première dans un article d'une ordonnance du comte d'Armagnac donnée à Toulouse le 21 octobre 1356 (Ordonnances des roys de France de la troisième race, recueillies par ordre chronologique..., t. III, p. 99-110).

15. Voir la section 2.4 de notre thèse de doctorat («La réponse des États », p. 118-138).

16. Cahier des États réunis à Mazères en octobre 1381 (Archives municipales de Narbonne, AA 177).

17. Ibid.

18. Paul Dognon, Quomodo tres status Linguae Occitanae, ineunle $X V^{\circ}$ saeculo, inter se convenire assueverint, Toulouse : Privat, 1896, p. 63-64.

19. Cahier des États réunis à Toulouse en juillet 1419 (Henri Gilles, Les États de Languedoc au XVe siècle, op. cit., p. -j. $\left.\mathrm{n}^{\circ} 1\right)$.

20. En 1469-1470, quelques territoires du Languedoc, dont la jugerie de Rivière et Verdun, sont rattachés à l'apanage de Charles de France. Les États n'auront de cesse, en vain, d'en revendiquer le retour. Voir le cahier des États réunis à Montpellier en juin 1479 (Archives départementales de l'Ardèche, C 697, $\mathrm{n}^{\circ}$ 10).

21. Albert Rigaudière, Gouverner la ville au Moyen Âge, Paris : Anthropos-Economica, 1993, p. 78.

22. Dans le même ordre d'idée, Lydwine Scordia a rappelé l'importance de ces deux aspects l'obéissance et l'amour - dans les relations entre le roi et les sujets, allant jusqu'à affirmer que c'est par amour que l'obéissance est "volontaire et librement consentie ». Voir Id., "Le roi doit vivre du sien». La théorie de l'impôt en France (XIIIe-XVe siècles), Paris: Institut d'études augustiniennes, 2005, p. 391.

23. Philippe Contamine, «La royauté française et le pays de langue d'oc», in: Philippe Contamine (dir.), Hommes et terres du Sud. Structures politiques et évolution des sociétés, XIe-XVIIIe siècle. Actes du 126e congrès des sociétés historiques et scientifiques (Toulouse, 2001), Paris : Éditions du Comité des travaux historiques et scientifiques, 2009, p. 57.

24. Patrick Arabeyre, Les Idées politiques à Toulouse à la veille de la Réforme. Recherches autour de l'œuvre de Guillaume Benoît (1455-1516), Toulouse : Presses de l'Université des sciences sociales de Toulouse, 2003, p. 67 et suiv.

25. Philippe Contamine, «La royauté française et le pays de langue d'oc », art. cit. , p. 217.

26. Philippe Wolff (éd.), Histoire du Languedoc, nouv. éd., Toulouse : Privat, 2000, p. 225.

27. Henri Bresc, « La démocratie dans les communautés et dans l'État en France méridionale et en Provence aux derniers siècles du Moyen-Âge ", in : Actes de l'Université d'été 2002-2003, textes 
réunis par Jòrgi Peladan, Nîmes: Maison pour l'Animation et la Recherche Populaire Occitane/ Institut d'Estudis Occitans 30, 2005, p. 20.

28. Philippe Contamine, "La royauté française et le pays de langue d'oc », art. cit. , p. 207.

29. Thomas N. Bisson, Assemblies and Representation in Languedoc in the Thirteenth Century, Princeton (N.J.) : Princeton University Press, 1964, p. 297.

30. Léonard Dauphant, Le Royaume des quatre rivières : L'espace politique français (1380-1515), Seyssel : Champ Vallon, 2012.

\section{AUTEUR}

\section{SYLVIE QUÉRE}

(Université du Québec, Montréal) 